\title{
Reconfigurable swarms of nematic colloids commanded by photoactivated surface patterns
}

\author{
Sergi Hernàndez-Navarro, ${ }^{[\mathrm{a}],[\mathrm{dd}]}$ Pietro Tierno, ${ }^{[\mathrm{b}],[\mathrm{d}]}$ Joan Anton Farrera, ${ }^{[\mathrm{c}][\mathrm{d}]}$ Jordi Ignés- \\ Mullol, ${ }^{*}\left[\mathrm{a},[\mathrm{d}]\right.$ and Francesc Sagués ${ }^{[\mathrm{a}],[\mathrm{d}]}$
}

\begin{abstract}
Different phoretic driving mechanisms have been proposed for the transport of solid or liquid microscopic inclusions in integrated chemical processes. Here we show that a substrate chemically modified with photosensitive self-assembled monolayers allows to directly command the assembly and transport of large ensembles of micron-size particles and drops dispersed in a thin layer of anisotropic fluid. Our strategy separates particle driving, realized via $A C$ electrophoresis, and steering, achieved by elastic modulation of the host nematic fluid. Inclusions respond individually or in collective modes following arbitrary reconfigurable paths imprinted via UV/blue illumination. Relying solely on generic material properties, the proposed procedure is versatile enough for the development of applications involving either inanimate or living materials.
\end{abstract}

In the emerging field of microfluidics, analytic operations are usually performed by pumping chemicals through channels that are typically hundreds of micrometers wide ${ }^{[1]}$. Versatility of integrated lab-on-a-chip devices demands the ability to transport femtosized volumes of solid ${ }^{[2]}$ or liquid ${ }^{[3]}$ chemical cargo without relying on permanent geometrical constraints. Colloidal suspensions, formed by microsized inclusions embedded in a carrier fluid are a natural starting point for such a strategy. The self-assembly of colloidal entities can be tuned by controlling inclusion size ${ }^{[4]}$ and shape $e^{[5]}$, surface chemistry ${ }^{[6]}$, or by suspending the colloids in a structured liquid ${ }^{[7]}$.

Different strategies exist to drive individual colloids dispersed in a liquid ${ }^{[8]}$, although the control and large-scale addressability of collections of motile inclusions has proven challenging to realize. Holographic optical traps allow to achieve direct control over the placement of a few hundreds of inclusions $^{[8 \mathrm{a}]}$, although the technique is limited by the field of

[a] S. Hernàndez-Navarro, Prof. J. Ignés-Mullol, Prof. F. Sagués Department of Physical Chemistry

Universitat de Barcelona

Martí i Franquès 1, 08028 Barcelona, Catalonia, Spain.

E-mail: jignes@ub.edu

[b] Dr. P. Tierno

Department of Structure and Constituents of Matter

Universitat de Barcelona

Avinguda Diagonal 647, 08028 Barcelona, Catalonia, Spain.

[c] Prof. J. A. Farrera

Department of Organic Chemistry

Universitat de Barcelona

Martí i Franquès 1, 08028 Barcelona, Catalonia, Spain.

[d] S. Hernàndez-Navarro, Dr. P. Tierno, Prof. J. A. Farrera, Prof. J. Ignés-Mullol, Prof. F. Sagués

Institut de Nanociència i Nanotecnologia

Universitat de Barcelona

Martí i Franquès 1, 08028 Barcelona, Catalonia, Spain.

Supporting information for this article is given via a link at the end of the document view of the optical system. Reconfigurable colloidal clusters can be achieved through the interplay between phoretic and osmotic mechanisms ${ }^{[9]}$, which has opened new perspectives for selfassembly ${ }^{[10]}$ and dynamic transport of inclusions ${ }^{[11]}$ or swarms ${ }^{[\mathrm{ib}}$, 12].

Of particular interest is the use of alternating current (AC) electric fields, which avoid the ion migration mechanisms caused by direct current driving. While individual metallo-dielectric Janus particles can be driven by this method in aqueous media ${ }^{[13]}$, nematic liquid crystal (NLC) hosts ${ }^{[14]}$ allowed to use AC electrophoresis to transport solid ${ }^{[15]}$ or liquid inclusions ${ }^{[16]}$. A NLC is an oil featuring long-range orientational order (the director field) of its constituent molecules, but can also be realized in concentrated aqueous suspensions of amphiphiles (lyotropic liquid crystals).$^{[14]}$ One of the most intriguing characteristics of these viscoelastic materials is their interaction with non-flat surfaces, in particular colloidal inclusions, that leads to the proliferation of topological point and line defects. This can result in colloid-based self-assembling materials ${ }^{[17]}$ and can be used as a positioning mechanism for embedded colloids ${ }^{[18]}$.

We integrate all the above possibilities in a novel electrooptical technique that permits to remotely address the reversible assembly and collective transport of micron-size colloidal inclusions of arbitrary shape and composition, both solid and liquid, dispersed in a thin NLC film. We use liquid crystalenabled electrophoresis (LCEEP) $)^{[15 b]}$ to propel the colloidal inclusions embedded in a NLC cell, and a photosensitive anchoring layer to modify the local director field, which, in turn, sets the direction of colloidal motion. We assemble or disassemble swarms, and control their placement and motion over arbitrary paths on a surface.

Our system consists of a NLC film inserted between parallel glass plates coated with a transparent electrode, which allows application of electric fields perpendicular to the plates. One of the plates is functionalized with an azosilane photosensitive self-assembled monolayer that allows to alternate between perpendicular (homeotropic) or planar (tangential) boundary conditions (anchoring) of the contacting NLC. The counter plate is treated with a spin-coated polyimide compound to achieve strong and permanent homeotropic anchoring. Without external influences, these boundary conditions lead to uniform homeotropic anchoring of the NLC. By irradiating with UV $(365 \mathrm{~nm})$ light from an incoherent source we force the azosilane monolayer to adopt the cis (planar) configuration, which can be easily reverted to trans (homeotropic) when using blue $(455 \mathrm{~nm})$ illumination (Figure 1 and Figure S1). We employ a NLC with negative dielectric anisotropy, which aligns parallel to the glass plates upon application of the electric field. This leads to degenerate planar alignment conditions, since all in-plane orientations for the director field are energetically equivalent, thus enabling the local addressability of the NLC director. 


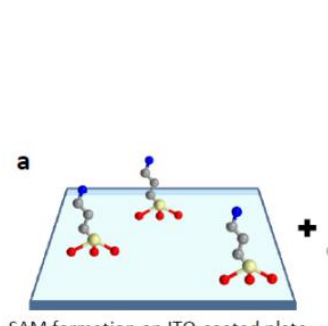

SAM formation on ITO-coated plate

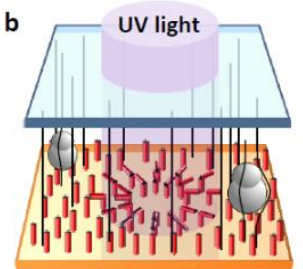

Photomodulation of a nematic colloid layer

Figure 1. Schematics showing the preparation of photo-addressable nematic colloids. (a) Representation of the two-step surface functionalization to prepare a photosensitive ITO coated glass surface. The grafted alkylazobenzene chains can be reversibly switched between the cis and trans isomers. (b) Colloids are dispersed in a nematic liquid crystal confined between a photosensitive and a non-photosensitive plate. This allows to reversibly light-scribe patterns of in-plane alignment (here, a circular spot characterized by a radial pattern). The embedded anisometric particles are aligned by the local nematic director (black lines). Upon application of an AC electric field (c) planar regions expand, and the particles are electrophoretically driven along the local director.

As colloidal inclusions we use pear-shaped microparticles made of polystyrene, a material that promotes planar orientation of the NLC on the particle surface (Experimental Section and Section S1). The chosen particle shape guarantees a dipolar component in the distortion of the local director field, a requirement for LCEEP to be an efficient propulsion mechanism. Earlier evidences of this mechanism have employed spherical solid $^{[15 a]}$ or liquid ${ }^{[16]}$ inclusions, relying on surface functionalization to achieve a homeotropic anchoring on the particle surface, which leads to a dipolar arrangement of the NLC director around the inclusion. However, homeotropic anchoring has the disadvantage that it can degenerate into a quadrupolar director configuration for which LCEEP propulsion is not effective. Our choice thus ensures that all particles in a large ensemble are similarly propelled.

In the absence of irradiation or electric field, particles will be oriented perpendicularly to the cell plates, following the uniform NLC director. Shinning the cell for a few seconds with a spot of UV light (Figure S2) forces the NLC in contact with the azosylane-treated surface to transit to a planar configuration. As shown in Figure 2a and Figure S3, this configuration conforms to the applied irradiation (Figure S6) by locally adopting a splay (radially-spread) texture organized around a central defect ${ }^{[14]}$. Application of an external AC field forces the bulk NLC to adopt the splay configuration that now extends for several millimeters, well beyond the size of the irradiated spot, thanks to the anchoring degeneracy. The induced configuration is stable for days under $\mathrm{AC}$ field, well past the half-life for thermal relaxation of the azosilane film, which is about 30 minutes (Figure S7).

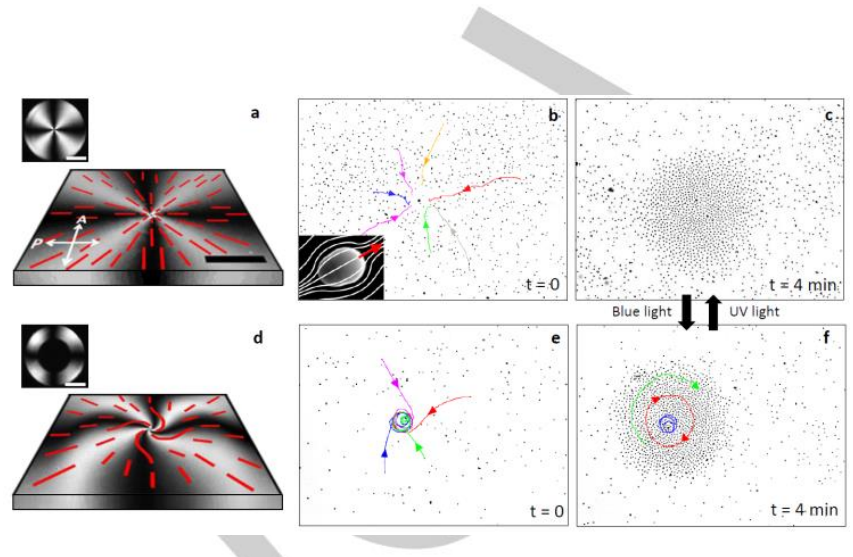

Figure 2. Formation of nematic colloidal clusters. Top (a-c) [bottom, (d-f)] row of images illustrating the formation of a colloidal aster (vortex). (a) and (d) are images between crossed polarizers of the imprinted NLC texture leading to a cross (a) or a spiral (d) attracting pattern. Dashed red lines represent the NLC director orientation. The insets show the planar photoaligned circle (inset a) or corona (inset d) prior to application of the electric field. Sequence b-c (e-f) shows the formation of an aster (vortex) after application of an electric field with frequency $10 \mathrm{~Hz}$ and amplitude $0.87 \mathrm{~V} / \mu \mathrm{m}$. The trajectories followed by several particles are superimposed to the images to illustrate the cluster formation mechanism. Inset in (b) shows a S.E.M. image of a single pearshaped particle $(3 \mu \mathrm{m} \times 4 \mu \mathrm{m})$, with NLC field lines and the direction of motion The colloidal aster in (c) and vortex in (f) can be interconverted by suitable irradiation protocols, as explained in the text. The scale bars are $200 \mu \mathrm{m}$ for all images and $500 \mu \mathrm{m}$ for the two insets (a and d).

The region with radial alignment will be the basin of attraction for dispersed particles, which tumble instantaneously following the NLC director so that their long axis lays, on average, parallel to the cell plates (Figure S3). Simultaneously, LCEEP sets the particle into motion at a constant speed. This feature allows, for instance, to decouple NLC realignment and particle motion, since their speed is negligible for field frequencies above $50 \mathrm{~Hz}$, while NLC realignment can be achieved even for frequencies in the $\mathrm{kHz}$ range and above ${ }^{[14]}$. All particles move following the local NLC director, with roughly half of them being attracted by the photoinduced radial defect and half of them being repelled by it. This is a consequence of the random tumbling of the particle dipole from their initial orientation perpendicular to the planar alignment induced by the AC field (Figure S4). As shown in Figure 2b-c (Movie S1), particles follow the NLC field lines and assemble into a growing aster-like configuration where they jam and become arrested.

We can easily switch the assembling mode between an arrested aster and a rotating vortex cluster by taking advantage of the elastic properties of the dispersing NLC, and the fact the particles are slave to the NLC director field. This is achieved by erasing the central region of an imprinted UV area with a smaller spot of blue light, which forces the cis to trans isomerisation leading to homeotropic NLC alignment. 

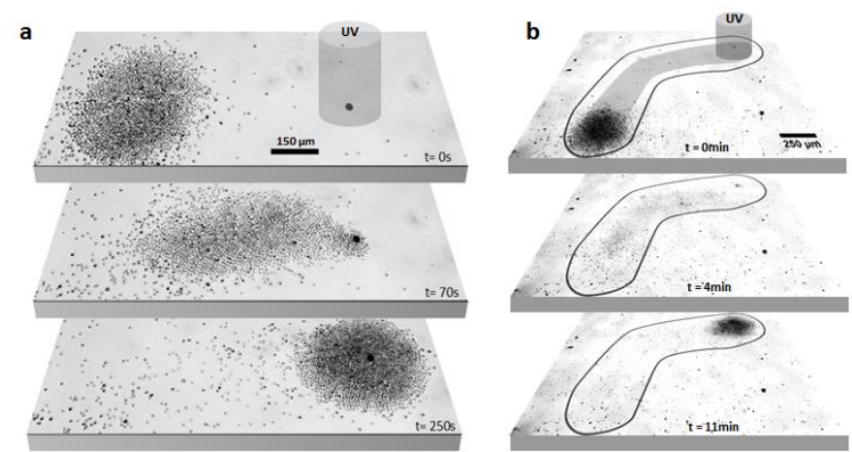

Figure 3. Cluster addressability. Image sequence of a particle swarm traveling across the LC cell due to in situ reconfiguration of the NLC field. In (a), The photoaligned spot initially centered with the cluster is moved $600 \mu \mathrm{m}$ to the right in a straight, as indicated by the dark spot. In (b) a longer hybrid track combining curved and straight segments is demonstrated. The contour of the track, only visible between crossed polarizers, is outlined. The applied sinusoidal electric field has amplitude of $0.74 \mathrm{~V} / \mu \mathrm{m}$ and a frequency of $10 \mathrm{~Hz}$.

As shown in Figure $2 \mathrm{~d}$ and inset, upon application of the AC field the planar alignment in the resulting circular corona is extended both outwards and inwards. The region surrounding the inner defect now features degenerate planar anchoring conditions, so it relaxes from the pure splay texture to the less energy demanding bend (rotational) texture. ${ }^{[19]}$ As a consequence, particles follow spiral trajectories, and assemble into a rotating vortex, preceding around the central defect with a constant linear velocity, as seen in Figure $2 \mathrm{e}, \mathrm{f}$ (Movie S1). The vortex cluster can be transformed back into an arrested aster by irradiating the region with a UV light spot. Both assembly modes can thus be reversibly interconverted in real time via photoelastic control (Movie S1).

The reversibility and quick response of the photoalignment layer enables straightforward cluster addressability. A preformed aggregate of arbitrary size, either aster or vortex-like, can be relocated to a pre-designed place anywhere within the experimental cell with minimum dismantlement of the cluster structure by changing the location of the UV spot. An example of this process is shown in Figure 3a (also Movie S2 and Figure S4). After blocking the LCEEP mechanism by increasing the field frequency above $50 \mathrm{~Hz}$, the center of attraction is translated $600 \mu \mathrm{m}$ to the right. Once LCEEP is reactivated, the swarm of particles moves towards the new position developing a leading edge around which the particles assemble. Alternatively, by the same principle one can imprint predesigned arbitrary paths connecting distant locations inside the cell or draw circuits with complex topologies as a simple way to accumulate colloidal swarms in the irradiated area and further entrain them collectively as shown in Figure 3b (Movie S2).

Our strategy to command colloidal aggregation can be readily implemented for the direct or indirect transport of chemical cargo in a channel-free microfluidic environment. For instance, one can build photoactivated lattices of clusters with arbitrary symmetry on an extended surface by imprinting the corresponding distribution of irradiated spots. As an example, in Figure 4a (Movie S3) a triangular lattice of UV spots is imprinted onto the photosensitive surface. Upon application of the electric field, these spots compete as attractors for the particles dispersed in the NLC.

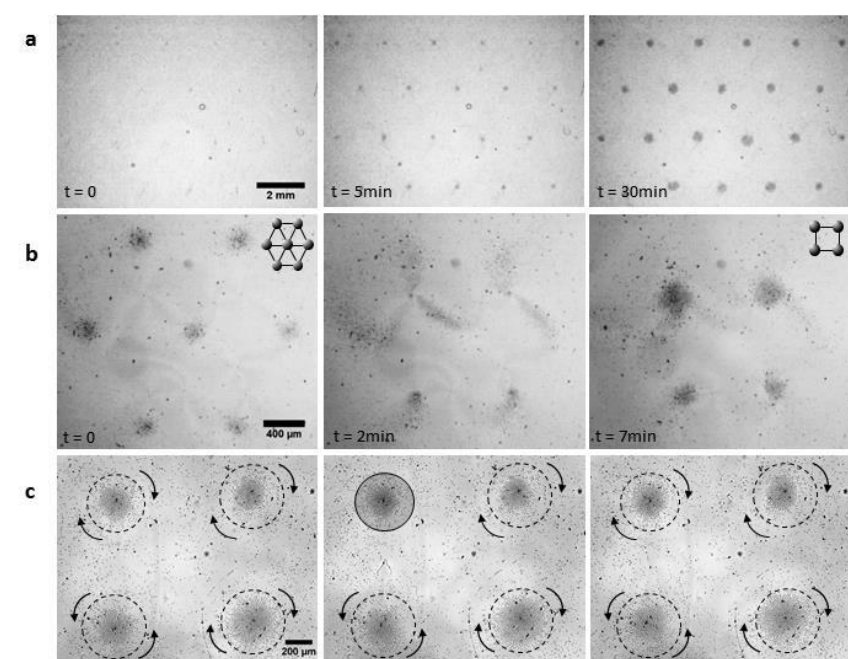

Figure 4. Reconfigurable cluster lattices. (a) Image sequence of the formation of a triangular lattice of particle clusters. A sinusoidal electric field of $0.74 \mathrm{~V} / \mu \mathrm{m}$ and $10 \mathrm{~Hz}$ is used to drive the particles. (b) Image sequence of the transformation from a triangular to a square lattice. The original distribution of attractor spots is erased with blue light $(455 \mathrm{~nm})$, and a square lattice is subsequently imprinted using UV light $(365 \mathrm{~nm})$. The cluster rearrangement occurs when a sinusoidal electric field of $0.78 \mathrm{~V} / \mu \mathrm{m}$ and $10 \mathrm{~Hz}$ is applied. Swarms of particles subsequently form and redistribute into the reconfigured lattice. (c) The dynamic state of clusters on a lattice can be individually addressed. While all the clusters are in a rotating dynamics, the top left cluster is first arrested and then restarted.

Rearrangement of the entire lattice readily achieved by conveniently reshaping the pattern of projected light spots. We demonstrate this addressability in Figure 4b (Movie S3), where the initially triangular lattice is transformed into a square one. Swarms of particles redistribute in the NLC cell according to the new director landscape. Once the electric field is switched off, the instantaneous pattern of clusters is preserved for an extended amount of time. For the used NLC, with a dynamic viscosity $\eta \simeq 0.1 \mathrm{~Pa} \mathrm{~s}$, we estimate a self-diffusion coefficient $D$ $\simeq 10^{-3} \mu \mathrm{m}^{2} \mathrm{~s}^{-1}$. Thus, it would take several hours for particles of the size used here to diffuse a few microns, and the spontaneous disaggregation of a cluster could take months.

We can also take advantage of our ability to remotely control the dynamic state of an individual cluster to enable complex mixing patterns in a confined chemical system. As demonstrated for a single aggregate, equally any site in a lattice can be reversibly switched between an arrested aster or a rotating vortex of particles. As example, we show in Figure 4c (Movie S3) the remote control of just one of the clusters in a square lattice. We arrest the dynamics on this cluster via a spot of UV light, while neighboring clusters remain unaffected. This shows our ability to selectively address a single swarm, and thus to implement arbitrarily complex local mixing patterns.

The reported swarming behavior can be employed to actuate on larger embedded objects for which electrophoretic propulsion is not effective. In Figure 5a (Movie S4), a large glass cylinder is set into rotational motion due to the collective interaction with the particles in a vortex, which has been previously nucleated on the cylindrical inclusion. The angular speed of the embedded object can be tuned by varying the speed of the driven particles, which is controlled by means of the applied AC field. 


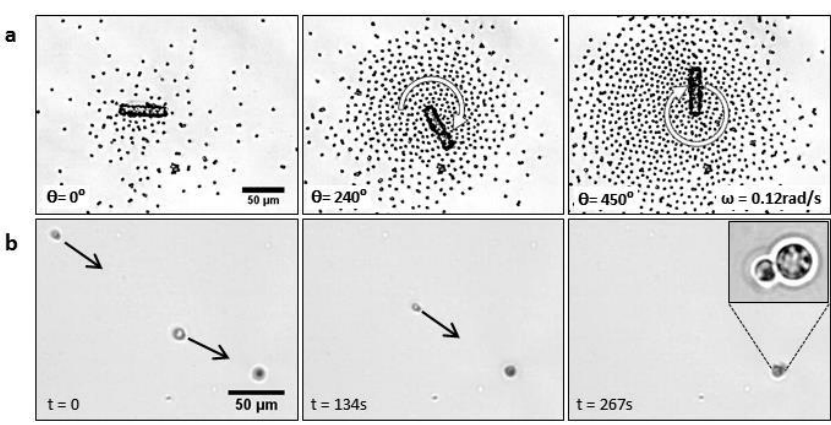

Figure 5. Microfluidic applications. (a) A glass microrod (50 $\mu \mathrm{m}$ long, $10 \mu \mathrm{m}$ in diameter) embedded in a microparticle swarm is set into rotation by the action of the surrounding inclusions. (b) Glycerol droplets are accumulated on a spot. Two droplets aggregated this way are shown in the magnified inset.

Inclusions of any nature are susceptible to be transported using the strategies described here, with the sole requirement that the NLC director has dipolar symmetry around the colloids. In the case of liquid inclusions, which will spontaneously adopt a spherical shape, the presence of an adsorbed surfactant can be employed to adjust the NLC anchoring and obtain the necessary configuration. In Figure 5b (Movie S4) we demonstrate the controlled aggregation of femtosized volumes of glycerol (stabilized with Sodium Dodecylsulphate) into an arbitrary spot. Droplets within a designed basin of attraction can be temporarily stored on a defined location for further processing.

The underlying physicochemical mechanisms rely on generic properties of the involved materials, and can be employed to establish complex local mixing patterns or drive larger embedded objects. Furthermore, since liquid-crystal enabled AC electrophoresis is also a valid transport mechanism for liquid inclusions, we can envisage an extension of the reported strategy to living systems by employing biocompatible lyotropic liquid crystals ${ }^{[20]}$. Although biocompatible catalytic micropumps have recently been demonstrated ${ }^{[21]}$, the perspective of control and addressability offered by our strategy are unparalleled. Finally, and on a broader perspective, the ability to control the dynamic assembly of colloidal inclusions over a surface can lead to novel devices, and can be employed to obtain model systems to study swarming behavior or the dynamics of soft active matter ${ }^{[22]}$.

\section{Experimental Section}

Nematic liquid crystal (NLC) cells were prepared using $0.7 \mathrm{~mm}$ thick microscope slides of size $15 \times 25 \mathrm{~mm}^{2}$, coated with a thin layer of indium-tin oxide (ITO) with a surface resistivity of $100 \Omega$ per square (VisionTek Systems). The plates were cleaned by sonication in a $1 \%$ Micro-90 (Sigma-Aldrich) solution, rinsed with ultrapure water $\left(18.2 \mathrm{M} \Omega \cdot \mathrm{cm}\right.$, Millipore Milli-Q Gradient A-10), and dried at $110^{\circ} \mathrm{C}$ for $20 \mathrm{~min}$. To prepare photosensitive plates (Supplementary Section 1), the ITO surface was first coated with a self-assembled monolayer of (3-aminopropyl)triethoxysilane (APTES, Sigma-Aldrich) ${ }^{[23]}$. The surface was subsequently functionalized with the azobenzene 4octyl-4'-(carboxy-3-propyloxy)azobenzene $(8 \mathrm{Az} 3 \mathrm{COOH}){ }^{[24]}$. The synthesis was performed in a dimethylformamide medium (peptide synthesis grade, Scharlau) through an amide group formation between the amino terminal group of APTES and the acid group of
$8 \mathrm{Az} 3 \mathrm{COOH}$, using pyBOP (>97\%, Fluka) as coupling agent, at a molar ratio $1: 1.25$ (8Az3COOH:pyBOP). We measured UV-vis spectra in order to verify the photosensitivity of the resulting azosilane film (Supplementary Figure S5). To prepare counterplates with homeotropic anchoring, the hydrophilic APTES-coated surfaces were spin-coated with a polyimide compound (0626 from Nissan Chemical Industries, using a 5\% solution in the solvent 26 also from Nissan) at $2000 \mathrm{rpm}$ for $10 \mathrm{~s}$, prebaked $1 \mathrm{~min}$ at $80^{\circ} \mathrm{C}$ to evaporate the solvent and then cured $45 \mathrm{~min}$ at $170^{\circ} \mathrm{C}$. A photosensitive and a non-photosensitive plate were then separated by a polyethylene terephtalate film (Mylar, Goodfellow, nominal thickness 13 and 23 $\mu \mathrm{m})$ and glued together along two sides with the ITO layers facing inwards.

Polystyrene anisometric particles (pear-shaped, $3 \times 4 \mu \mathrm{m}$, Magsphere) were dispersed in a nematic liquid crystal with negative dielectric anisotropy (MLC-7029, Merck, room temperature properties: $\left.\Delta \varepsilon(1 \mathrm{kHz})=-3.6, \mathrm{~K}_{1}=16.1 \mathrm{pN}, \mathrm{K}_{3}=15.0 \mathrm{pN}\right)$. Cells were filled with freshly prepared dispersions by capillary action and sealed with glue.

Irradiation of the samples at wavelengths $365 \mathrm{~nm}$ and $455 \mathrm{~nm}$ was performed by means of a custom-built LED epi-illumination setup integrated in an optical microscope. Typical light power densities were $0.1 \mathrm{~W} / \mathrm{cm}^{2}$ with a spot size in the sub-millimeter range (Supplementary Section S2). Sinusoidal electric fields were applied using a function generator and an amplifier. Amplitudes were in the range 0 to $35 \mathrm{~V}$ peak to peak, while frequencies for particle motion were in the range 3 to $50 \mathrm{~Hz}$.

\section{Acknowledgements}

We thank Patrick Oswald for the polyimide compound. We acknowledge financial support by MICINN (Project numbers FIS2010-21924C02, FIS2011-15948-E) and by DURSI (Project no. 2009 SGR 1055). S.H.-N. acknowledges the support from the FPU Fellowship (AP2009-0974). P.T. further acknowledges support from the ERC starting grant "DynaMO" (No. 335040) and from the "Ramon y Cajal" program (No. RYC-2011-07605).

Keywords: colloids $\bullet$ self-assembly $\bullet$ microreactors $\bullet$ liquid crystals • electrophoresis

[1] a) E. Kim, Y. Xia, G. M. Whitesides, Nature 1995, 376, 581-584; b) T. Squires, S. Quake, Reviews of Modern Physics 2005, 77, 977-1026.

[2] L. Baraban, D. Makarov, R. Streubel, I. Monch, D. Grimm, S. Sanchez, O. G. Schmidt, ACS nano 2012, 6, 3383-3389.

[3] H. Song, J. D. Tice, R. F. Ismagilov, Angew Chem Int Ed Engl 2003, 42, 768-772.

[4] A. Yethiraj, J. H. J. Thijssen, A. Wouterse, A. van Blaaderen, Advanced Materials 2004, 16, 596-600.

[5] S. C. Glotzer, M. J. Solomon, Nature materials 2007, 6, 557-562.

[6] a) S. Jiang, Q. Chen, M. Tripathy, E. Luijten, K. S. Schweizer, S. Granick, Adv Mater 2010, 22, 1060-1071; b) Y. Wang, D. R. Breed, V. N. Manoharan, L. Feng, A. D. Hollingsworth, M. Weck, D. J. Pine, Nature 2012, 491, 51-55.

[7] P. Poulin, H. Stark, T. C. Lubensky, D. A. Weitz, Science 1997, 275, 1770-1773.

[8] a) D. G. Grier, Nature 2003, 424, 810-816; b) W. F. Paxton, K. C Kistler, C. C. Olmeda, A. Sen, S. K. St Angelo, Y. Cao, T. E. Mallouk, P. E. Lammert, V. H. Crespi, Journal of the American Chemical Society 2004, 126, 13424-13431; c) W. F. Paxton, P. T. Baker, T. R. Kline, Y. Wang, T. E. Mallouk, A. Sen, Journal of the American Chemical Society 2006, 128, 14881-14888; d) J. Howse, R. Jones, A. Ryan, T. Gough, R. Vafabakhsh, R. Golestanian, Physical Review Letters 2007, 99, 048102; e) J. Wang, ACS nano 2009, 3, 4-9; f) I. Buttinoni, J. Bialké, F. Kümmel, H. Löwen, C. Bechinger, T. Speck, Physical Review Letters 2013, 110, 238301; g) W. Wang, T. Y. Chiang, D. Velegol, T. E. 
Mallouk, Journal of the American Chemical Society 2013, 135, 1055710565; h) W. Gao, A. Pei, R. Dong, J. Wang, Journal of the American Chemical Society 2014, 136, 2276-2279.

[9] a) W. Duan, R. Liu, A. Sen, Journal of the American Chemical Society 2013, 135, 1280-1283; b) M. Ibele, T. E. Mallouk, A. Sen, Angew Chem Int Ed Engl 2009, 48, 3308-3312; c) J. Palacci, S. Sacanna, A. P. Steinberg, D. J. Pine, P. M. Chaikin, Science 2013, 339, 936-940.

[10] A. Bricard, J. B. Caussin, N. Desreumaux, O. Dauchot, D. Bartolo, Nature 2013, 503, 95-98.

[11] J.-B. Caussin, A. Solon, A. Peshkov, H. Chaté, T. Dauxois, J. Tailleur, V. Vitelli, D. Bartolo, Physical Review Letters 2014, 112, 148102.

[12] a) D. Kagan, S. Balasubramanian, J. Wang, Angew Chem Int Ed Eng 2011, 50, 503-506; b) A. A. Solovev, S. Sanchez, O. G. Schmidt, Nanoscale 2013, 5, 1284-1293.

[13] S. Gangwal, O. Cayre, M. Bazant, O. Velev, Physical Review Letters 2008, 100, 058302.

[14] P. Oswald, P. Pieranski, Nematic and cholesteric liquid crystals : concepts and physical properties illustrated by experiments, Taylor \& Francis, Boca Raton, 2005.

[15] a) O. D. Lavrentovich, I. Lazo, O. P. Pishnyak, Nature 2010, 467, 947 950; b) O. D. Lavrentovich, Soft Matter 2014, 10, 1264-1283.

[16] S. Hernàndez-Navarro, P. Tierno, J. Ignés-Mullol, F. Sagués, Soft Matter 2013, 9, 7999-8004.
[17] a) I. Musevic, M. Skarabot, U. Tkalec, M. Ravnik, S. Zumer, Science 2006, 313, 954-958; b) T. A. Wood, J. S. Lintuvuori, A. B. Schofield, D Marenduzzo, W. C. Poon, Science 2011, 334, 79-83.

[18] A. Martinez, H. C. Mireles, Smalyukh, II, Proceedings of the National Academy of Sciences of the United States of America 2011, 108, 20891-20896.

[19] For this material, the bend elastic constant is smaller than the splay elastic constant. Therefore, in the absence of boundary conditions bend distortions will be favored.

[20] S. Zhou, A. Sokolov, O. D. Lavrentovich, I. S. Aranson, Proceedings of the National Academy of Sciences of the United States of America 2014, 111, 1265-1270.

[21] S. Sengupta, D. Patra, I. Ortiz-Rivera, A. Agrawal, S. Shklyaev, K. K Dey, U. Cordova-Figueroa, T. E. Mallouk, A. Sen, Nature chemistry 2014, 6, 415-422.

[22] M. C. Marchetti, J. F. Joanny, S. Ramaswamy, T. B. Liverpool, J. Prost, M. Rao, R. A. Simha, Reviews of Modern Physics 2013, 85, 1143-1189.

[23] J. A. Howarter, J. P. Youngblood, Langmuir 2006, 22, 11142-11147.

[24] J. Crusats, R. Albalat, J. Claret, J. Ignés-Mullol, F. Sagués, Langmuir 2004, 20, 8668-8674. 


\section{Entry for the Table of Contents}

\section{COMMUNICATION}

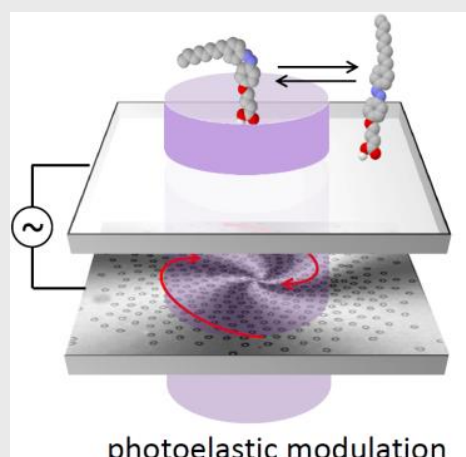

photoelastic modulation

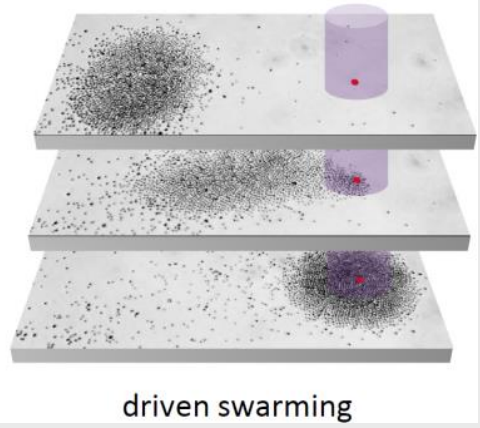

driven swarming
Sergi Hernàndez-Navarro, Pietro Tierno, Joan Anton Farrera, Jordi Ignés-Mullol*, and Francesc Sagués

Page No. - Page No.

Reconfigurable swarms of nematic colloids commanded by photoactivated surface patterns

Swarming into focus. We present a novel strategt to directly command the assembly and transport of large ensembles of colloidal particles or droplets dispersed in a film of anisotropic fluid, which enables to drive the inclusions by means of AC electrophoresis. Individual or collective particle steering is independently achieved by elastic modulation of the host nematic along reconfigurable paths on a photosensitive confining surface. 